\title{
L'orientation scolaire et professionnelle des filles : des « choix de compromis » ? Une enquête auprès de jeunes femmes issues de familles nombreuses
}

Girls' educational and occupational choices: are they "compromise-choices"? Research on young women from large families

Martine Court, Julien Bertrand, Géraldine Bois, Gaële Henri-Panabière et Olivier Vanhée

\section{OpenEdition \\ Journals}

Édition électronique

URL : http://journals.openedition.org/rfp/4212

DOI : $10.4000 /$ rfp.4212

ISSN : 2105-2913

Éditeur

ENS Éditions

Édition imprimée

Date de publication : 15 décembre 2013

Pagination : $29-40$

ISBN : 978-2-84788-521-7

ISSN : 0556-7807

\section{Référence électronique}

Martine Court, Julien Bertrand, Géraldine Bois, Gaële Henri-Panabière et Olivier Vanhée, « L'orientation scolaire et professionnelle des filles : des « choix de compromis »? Une enquête auprès de jeunes femmes issues de familles nombreuses ", Revue française de pédagogie [En ligne], 184 | 2013, mis en ligne le 15 décembre 2016, consulté le 10 décembre 2020. URL : http://journals.openedition.org/rfp/ 4212 ; DOI : https://doi.org/10.4000/rfp.4212 


\section{L'orientation scolaire et professionnelle des filles: des « choix de compromis»? Une enquête auprès de jeunes femmes issues de familles nombreuses}

\section{Martine Court, Julien Bertrand, Géraldine Bois, Gaële Henri-Panabière et Olivier Vanhée}

Dans les travaux sociologiques sur l'orientation, la notion de "choix de compromis » désigne la tendance qu'auraient les filles, plus que les garçons, à limiter leurs ambitions scolaires et professionnelles pour se diriger vers des métiers qui leur semblent faciles à concilier avec leurs futurs rôles familiaux. Cet article se propose d'analyser la portée de cette notion et ses présupposés, à partir d'un cas limite : celui de jeunes femmes issues de familles nombreuses, qui ont souvent vu leur mère confrontée de manière importante aux difficultés de la conciliation entre vie familiale et vie professionnelle. Constatant que les enquêtées qui subordonnent leur orientation scolaire et professionnelle à l'anticipation de ces difficultés sont en fait minoritaires, l'article interroge les conditions sociales de possibilité de ces " choix de compromis ».

Mots-clés (TESE) : orientation, choix des études, égalité des sexes, socialisation, famille, situation sociale.

Les recherches sur l'orientation ont mis en évidence depuis longtemps le poids du genre dans le choix des filières d'études (Vouillot, 2007). En 2004, au lycée, les filles sont surreprésentées dans les séries littéraires et médico-sociales, les garçons dans les filières scientifiques et techniques. Dans le supérieur, les filles sont majoritaires dans les études de droit, lettres et sciences humaines, dans les IUFM et dans les formations médicales et sociales ; les garçons le sont quant à eux dans les filières scientifiques et industrielles (Caillé, Lemaire \& Vrolant, 2002 ; Nauze-Fichet, 2005 ; Rosenwald, 2006). Si ces différences d'orientation résultent pour partie de logiques spécifiquement scolaires (notamment le sentiment qu'éprouvent les filles et les garçons d'être compétents dans telle ou telle matière ; Baudelot \& Establet,
1992), elles sont aussi le reflet d'aspirations professionnelles sexuellement différenciées (Stevanovic \& Mosconi, 2007). Interrogés sur le métier qu'ils souhaitent exercer, les lycéens garçons citent nettement plus souvent que les filles les professions d'ingénieur, d'informaticien ou de technicien, les filles les métiers du soin et de l'éducation. Les filles sont en particulier deux fois plus nombreuses que les garçons à vouloir être enseignantes (Bosse \& Guégnard, 2007).

Pour expliquer ces différences, M. Duru-Bellat a proposé, au début des années 1990, une analyse devenue classique, qui consiste à relier les choix d'orientation des jeunes filles à la façon dont elles envisagent leurs futurs rôles familiaux (Duru-Bellat, 1991, 1995, 2004). Selon cette analyse, si les filles et 
les garçons s'orientent vers des professions - et en amont vers des études - différentes, c'est avant tout parce qu'ils n'anticipent pas de la même façon les difficultés liées à l'articulation entre vie familiale et vie professionnelle. En l'occurrence, l'anticipation de ces difficultés conduit nombre de filles à faire ce que l'auteure nomme des " choix de compromis ", c'est-àdire à renoncer à des professions socialement valorisées, parce qu'elles les perçoivent comme incompatibles avec les contraintes d'une vie de famille, pour s'orienter vers des métiers moins reconnus, mais qui leur semblent plus faciles à concilier avec ces contraintes ${ }^{1}$. Au cours d'une enquête par entretiens réalisée en 1993 auprès d'une soixantaine d'élèves de première, M. Duru-Bellat (1995) observe ainsi que, dès l'âge du lycée, presque toutes les filles (et seulement une petite partie des garçons) s'interrogent sur la façon dont elles concilieront plus tard leur vie familiale et leur vie professionnelle, et que certaines d'entre elles choisissent leur métier et leur orientation en fonction de cette contrainte. D'un point de vue théorique, cette analyse se fonde sur le modèle de l'acteur rationnel (Boudon, 1979). Les " choix de compromis" sont présentés comme le produit d'un calcul entre les coûts et les bénéfices associés à différentes alternatives, par des actrices rationnelles ayant de "bonnes raisons " de faire ce qu'elles font ${ }^{2}$. En adoptant ce cadre théorique, M. Duru-Bellat souhaite éviter " l'écueil » consistant à " considérer les individus comme des marionnettes entièrement déterminées de l'extérieur » (Duru-Bellat, 1991, p. 258). II s'agit d'« accorder davantage de poids à la "logique de la situation" et donc au présent par rapport aux déterminations passées 》 (DuruBellat, 1995, p. 82).

Cette analyse des choix d'orientation des filles permet de montrer que les décisions d'ordre scolaire peuvent trouver leur principe dans des logiques qui débordent largement le cadre de l'école. Ce faisant, elle rappelle le rôle central que joue la sphère domestique dans la constitution des inégalités entre hommes et femmes. Cependant, la perspective théorique adoptée limite fortement l'analyse de ces " choix de compromis". En évacuant la question des " déterminations passées ", c'est-à-dire de la socialisation des jeunes filles, M. Duru-Bellat s'interdit en effet d'examiner et donc de mettre au jour les conditions sociales de possibilité de ces choix. Dès lors, les différences entre les jeunes filles qui opèrent ce type d'arbitrage et celles qui ne le font pas ne sont quasiment pas évoquées. La théorie du choix rationnel conduit à supposer que toutes les adolescentes évaluent de la même façon les coûts et les bénéfices associés à chaque choix et, avant même cela, que toutes réalisent effectivement une telle évaluation. $\mathrm{Si}$ M. Duru-Bellat note bien que la façon de mesurer les coûts et les bénéfices, comme le poids accordé à tel ou tel élément dans cette mesure, peuvent varier d'un individu à l'autre en fonction de ses propriétés sociales, elle n'examine pas empiriquement ce qui est au principe de cette variation, et passe donc sous silence les différences qui existent entre les jeunes filles de ce point de vue ${ }^{3}$.

L'intention de cet article est précisément d'analyser les conditions de possibilité de ces choix de compromis : chez quelles jeunes filles, dotées de quelles propriétés sociales, peut-on observer ce type de choix ? Dans quels cas ces choix sont-ils présents, dans quels cas sont-ils au contraire inexistants ? Ce projet suppose que l'on réintroduise dans l'analyse la question de la socialisation, largement évacuée par la théorie de l'acteur rationnel. Dans le cadre d'une sociologie dispositionnaliste (Bourdieu, 1979, 1980), soucieuse de mettre au jour les processus concrets à l'œuvre dans la fabrication sociale des individus (Lahire, 2000), on se propose ici d'examiner comment la socialisation familiale peut favoriser ou non ce type de choix. Nous souhaitons en particulier analyser comment cette façon de décider de ses choix d'orientation peut varier en fonction de la position que les filles occupent dans l'espace social ${ }^{4}$.

Nous nous fondons pour cela sur l'analyse d'une série d'entretiens réalisés avec des jeunes femmes âgées de 18 à 27 ans ayant terminé leurs études secondaires. Ces jeunes femmes ont été rencontrées au cours d'une enquête étudiant les parcours scolaires des enfants de familles nombreuses (Bertrand, Bois, Court et al., 2012)5. Cette population est particulièrement intéressante pour observer comment les filles peuvent être amenées à anticiper les difficultés de la conciliation entre rôles familiaux et professionnels, et comment cette préoccupation peut orienter leurs choix scolaires et professionnels. D'abord, les jeunes filles ayant grandi dans une famille nombreuse ont fréquemment pu voir leur mère confrontée de manière exacerbée aux difficultés de cette conciliation. Lorsqu'elles sont actives $-47 \%$ des mères de quatre enfants et plus le sont quand leurs enfants sont scolarisés (Blanpain, 2007) -, les mères de familles nombreuses cumulent en effet leur activité professionnelle avec une charge de travail domestique particulièrement lourde, non seulement parce qu'elles ont un nombre d'enfants élevé, mais aussi parce que la division sexuelle de ce travail au sein du couple est encore plus inégalitaire dans ces familles 
que dans les autres (Leturcq \& Wierink, 2009 ; Saint Pol \& Bouchardon, 2013). Ensuite, les filles issues d'une famille nombreuse souhaitent souvent - plus souvent que les autres - avoir un nombre relativement élevé d'enfants (Régnier-Loilier, 2006), et ce souhait peut amener une partie d'entre elles à se montrer particulièrement attentives à la façon dont elles concilieront plus tard leur vie familiale et leur vie professionnelle.
Dans la première partie de cet article, nous montrons que si l'anticipation des difficultés liées à la conciliation entre vie familiale et vie professionnelle peut effectivement jouer un rôle dans l'élaboration des choix d'orientation de certaines jeunes filles, cette logique ne s'observe en réalité que dans une minorité de cas. Nous nous attachons alors à mettre en lumière ce qui distingue, en termes de socialisation, celles qui prennent en compte cette dimension

Le matériau empirique sur lequel se fonde cet article est issu d'une enquête qualitative qui visait à analyser les effets de l'appartenance à une famille nombreuse sur les parcours scolaires des enfants. Alors que selon la définition administrative (pour la CNAF notamment), une famille «nombreuse " comprend trois enfants ou plus, les familles étudiées dans cette enquête en comptent toutes au moins quatre. La fixation de ce critère répond au souhait d'observer de manière en quelque sorte " grossie » des processus qui sont également à l'œuvre de façon moins visible dans les familles de trois enfants. La population d'enquête est composée de vingt-deux familles, «traditionnelles » ou recomposées, comprenant au moins un enfant âgé de moins de 30 ans ayant terminé ses études secondaires. Dans chaque famille, c'est le parcours scolaire de cet enfant-là qui a été étudié. Les analyses présentées ici portent sur les treize familles dans lesquelles cet enfant est une jeune fille.

Face à la difficulté d'accéder à une population relativement rare et non agrégée par une institution, le recrutement des enquêtés s'est fait principalement par interconnaissance. Afin d'éviter que cette interconnaissance ne vienne entraver le travail d'objectivation, les personnes qui ont conduit les entretiens ne connaissaient pas les personnes interviewées avant de les rencontrer. Lorsqu'un membre de l'équipe était proche d'une famille enquêtée, c'est toujours un autre enquêteur qui a réalisé les entretiens et les premières analyses. Nous nous sommes également efforcés de limiter les effets que peut produire ce mode de recrutement sur la composition de la population, en veillant à sélectionner des familles diversifiées du point de vue de leur position sociale. Au final, nous avons rencontré douze familles appartenant aux classes populaires (le père est ouvrier ou employé), cinq appartenant aux classes moyennes (le père est petit indépendant ou profession intermédiaire) et cinq aux classes supérieures (le père est cadre ou profession intellectuelle supérieure $)^{6}$. Sur les treize jeunes femmes interviewées, sept ont un père ouvrier, six ont un père membre des classes moyennes ou supérieures. Si nous avons réussi à constituer une population de familles diversifiée du point de vue des positions sociales, il a en revanche été plus difficile de faire varier les parcours scolaires des jeunes, la réussite scolaire des enfants favorisant l'acceptation des entretiens au sein des familles. La plupart des jeunes interrogés ont ainsi obtenu un diplôme supérieur ou égal au bac. Parmi les treize jeunes filles interviewées, une seule n'a pas le bac. Au moment de l'enquête, neuf sont étudiantes et quatre sont actives, dont deux au chômage.

Dans la majorité des cas, nous avons réalisé deux entretiens au sein de chaque famille, l'un avec l'« enfant », l'autre avec l'un de ses parents ou à défaut l'un-e de ses aîné-e-s. La plupart des personnes sollicitées sont apparues très désireuses de participer à l'enquête et de raconter leur expérience?. Pour beaucoup de parents, l'entretien était l'occasion de revendiquer la légitimité de leur mode de vie et notamment d'offrir une représentation positive d'eux-mêmes, se démarquant de l'image de parents « irresponsables », qui feraient des enfants sans penser à leur avenir. Pour les jeunes aussi, l'entretien permettait souvent d'offrir une image positive d'eux et de leur famille dans la mesure où la plupart d'entre eux ont connu des parcours scolaires de réussite. Dans quelques familles cependant, un seul entretien a pu être négocié, ou bien les entretiens ont été difficiles à conduire et n'ont pas pu être achevés. Dans la mesure où l'enquête portait sur le thème de l'école (elle était présentée comme « une étude sur la scolarité des enfants issus de familles nombreuses "), ces difficultés s'expliquent pour l'essentiel par le sentiment d'illégitimité scolaire éprouvé par certains jeunes et/ou par leurs parents. Nous les avons en effet rencontrées principalement auprès de jeunes (ou de parents de jeunes) de milieux populaires ayant connu des difficultés scolaires importantes et ne tenant vraisemblablement pas à revenir sur ces expériences.

De manière générale, les entretiens avaient pour but de reconstituer le parcours scolaire des jeunes interviewés ainsi que les conditions de leur socialisation familiale. Ils invitaient notamment ces jeunes (et leurs parents) à décrire comment s'était décidée leur orientation en fin de troisième, au lycée, puis le cas échéant après le bac. Les questions portant sur les éventuels projets professionnels étaient abordées à ce moment-là. Alors que, dans l'enquête de M. Duru-Bellat, la façon dont les enquêtés ont pris leurs décisions d'orientation à l'âge du lycée a été saisie au moment même où ces décisions se prenaient, dans notre propre enquête, elle a été reconstituée de manière rétrospective. Néanmoins, dans la plupart des cas, les jeunes interviewés ont été en mesure de nous livrer des récits précis de cet épisode de leur scolarité - survenu seulement quelques années auparavant. En outre, nous avons presque toujours pu compléter et croiser ces récits avec ceux d'un autre membre de la famille (un parent ou un-e aîné-e) ${ }^{8}$. 
de leur avenir dans l'élaboration de leurs choix d'orientation de celles qui ne le font pas. Enfin, nous nous centrons sur le cas - minoritaire - des jeunes filles qui subordonnent leur orientation à ce qu'elles anticipent de leurs futurs rôles familiaux, pour examiner dans quels cas cette manière de décider de son orientation constitue effectivement un choix " de compromis ", c'est-à-dire un choix impliquant objectivement un abaissement des ambitions scolaires et sociales des filles.

\section{SUBORDONNER SES CHOIX D'ORIENTATION SCOLAIRE À L'ANTICIPATION DES RÔLES FAMILIAUX : UNE CONDUITE MINORITAIRE}

Parmi les jeunes femmes interviewées, toutes celles qui ne sont pas encore actives souhaitent le devenir. Dans le même temps, presque toutes désirent avoir au moins deux enfants (la majorité souhaitant en avoir plus). Pour la plupart de ces jeunes femmes, la question de la conciliation entre leur future vie familiale et leur future vie professionnelle constitue donc potentiellement une préoccupation importante.

Cette préoccupation a orienté de manière décisive les choix scolaires et professionnels de certaines d'entre elles. Morgane Joubert9 (père ouvrier, mère secrétaire) envisage ainsi d'être institutrice dès l'âge du lycée parce qu'elle veut " un métier et des enfants " et qu'elle pense que cette profession offre des horaires de travail commodes pour une mère de famille. Après un bac ES, elle s'inscrit donc à l'université (en sociologie) afin d'obtenir une licence et de pouvoir se présenter au concours de professeur des écoles. Geneviève Lemaire (père radiologue, mère médecin du travail, tous deux à l'hôpital) a elle aussi choisi le métier d'enseignante parce qu'elle le pensait facile à concilier avec une vie de famille : " Je me disais: "Tu vas avoir des enfants, si tu veux rentrer tôt le soir, [t']en occuper, etc., prof c'est l'idéal. Au niveau des horaires, ce sera à peu près tranquille" ". Pendant son année de terminale (S), elle rencontre son futur mari, tous les deux parlent rapidement d'avoir des enfants (ils en veulent quatre ou cinq) et Geneviève s'inscrit en classe préparatoire scientifique dans le but d'intégrer une ENS. Elle poursuit ensuite ses études à l'université et obtient l'agrégation de mathématiques. Marion Bernier (père chef d'entreprise, mère kinésithérapeute), enfin, souhaite elle aussi, dès le lycée, avoir quatre ou cinq enfants et un métier qui permette "une vie de famille ». En seconde, elle envisage de devenir kinésithérapeute, et à sa mère qui l'incite plutôt à être médecin, elle répond que le métier de kinésithérapeute lui laisserait " plus de vie ». À l'issue du bac (S), elle s'inscrit finalement en faculté de médecine dans le but de devenir médecin. En deuxième année au moment de l'enquête, elle pense cependant s'orienter vers la médecine générale plutôt que vers la médecine spécialisée, et elle justifie à nouveau ce choix par son désir d'exercer un métier plus facile à concilier avec une vie de famille : «C'est très prenant [la médecine spécialisée], y'a beaucoup de gardes, alors pour avoir une vie de famille c'est un peu compliqué ».

Toutefois, à l'exception de ces trois jeunes femmes, aucune de nos enquêtées n'a fait ses choix d'orientation en fonction de cette logique. Au niveau du lycée, plusieurs disent ainsi s'être orientées "par hasard ", sans savoir pourquoi (" aucune idée ! ") et en n'ayant donc pas le sentiment de choisir réellement cette orientation. Plusieurs ont également choisi leur série de baccalauréat en fonction de logiques purement scolaires : rester dans l'enseignement général, ou s'inscrire en série $S$ parce qu'elles ont de bons résultats et que le choix de cette filière s'impose comme une évidence. Certaines enfin n'ont pas choisi leur orientation au lycée, mais se la sont vu imposer en raison de résultats scolaires jugés trop faibles ${ }^{10}$.

À l'issue du lycée, les choix d'orientation faits " par hasard " ou en fonction de logiques purement scolaires deviennent moins fréquents. Néanmoins, la plupart des enquêtées ne subordonnent toujours pas ces choix à des projets de vie familiale. Certaines choisissent leur filière d'études en suivant les injonctions qui leur sont adressées de manière explicite ou implicite par leurs parents. Héléna Chautard (père jardinier, mère aide-soignante), par exemple, s'inscrit en faculté de médecine sans conviction, en cédant aux incitations de ses parents qui rêvent d'avoir une fille médecin. D'autres, plus nombreuses, décident de leur orientation en fonction de leur intérêt pour une matière scolaire et non en fonction du désir d'exercer une profession en particulier. C'est le cas par exemple de Justine Wemel (père cadre, mère employée, tous les deux dans la fonction publique) qui, après avoir suivi une classe préparatoire littéraire pendant un an " pour réfléchir ", s'est inscrite en faculté de droit parce que cette formation l'intéressait. À la fin de sa première année, elle ne sait toujours pas ce qu'elle va " faire plus tard " mais elle souhaite continuer sa formation en droit parce qu'elle " aime les études". C'est également le cas de Bérangère Cotti (père 
ouvrier en invalidité, mère caissière), qui n'a jamais " rêvé » à aucun métier en particulier et qui est entrée aux Beaux-Arts uniquement par goût pour les arts plastiques. D'autres enquêtées, enfin, avaient une idée assez précise du métier qu'elles souhaitaient exercer lorsqu'elles ont décidé de leur orientation après le bac, mais elles n'ont pas choisi ce métier pour les facilités qu'il offre du point de vue de l'articulation entre vie familiale et vie professionnelle. Lorsqu'elles expliquent comment elles ont fait ce choix, elles n'évoquent pas spontanément le souhait d'exercer un métier qui laisse du temps pour une vie de famille. Aux relances des enquêteurs invitant à décrire ce qu'est un "bon métier » à leurs yeux, elles ne mentionnent pas la compatibilité avec la vie familiale.

Subordonner ses choix d'orientation à l'anticipation de ses futurs rôles familiaux apparaît donc comme une manière particulière de décider de son orientation, qui ne concerne pas la majorité des jeunes femmes rencontrées. Pour comprendre ce qui différencie les enquêtées de ce point de vue, il est nécessaire de comparer de manière précise la socialisation que les unes et les autres ont connue au cours de leur enfance et de leur adolescence.

\section{LES CONDITIONS DE LA SUBORDINATION DES CHOIX D'ORIENTATION À L'ANTICIPATION DES RÔLES FAMILIAUX}

\section{Pouvoir se projeter dans l'avenir : l'importance de l'origine sociale}

L'idée selon laquelle les filles subordonnent leurs projets d'orientation à l'anticipation des difficultés liées à la conciliation entre vie familiale et vie professionnelle suppose en premier lieu que celles-ci ont effectivement de tels projets. Or, comme de nombreux travaux l'ont établi, beaucoup de jeunes, notamment dans les milieux populaires, n'appréhendent pas leur orientation de cette façon (André, 2012 ; Dubet \& Martucelli, 1996 ; Orange, 2012 ; Rochex, 1992). Construire un " projet » d'orientation au sens que l'institution donne à ce mot $^{11}$ nécessite en effet un sentiment de maîtrise sur son avenir et une faculté à se projeter dans le temps long qui ne sont pas distribués de manière égale dans l'espace social. Alors que les parents de classes moyennes et supérieures possèdent fréquemment ces dispositions, qui leur permettent de développer des stratégies scolaires sur le long terme (van Zanten, 2009), les parents de milieux populaires - et leurs enfants manifestent plus souvent une forme d'« insouciance par rapport au futur » (André, 2012, p. 109), en lien avec une habitude de vivre au jour le jour et une valorisation forte du moment présent : " Cette logique rejoint ce que certains auteurs ont caractérisé comme "I'hédonisme" des classes populaires qui organisent moins leur trajectoire de manière rationnelle qu'elles ne le tablent sur la chance et les coups du destin12 (André, 2012, p. 44) ${ }^{13}$. Dans les fractions les plus précaires des classes populaires, les conditions matérielles d'existence réduisent tout particulièrement les possibilités de faire des projets. Se projeter dans l'avenir requiert en effet une stabilité matérielle minimale ainsi qu'une relative pérennité des revenus, qui seules permettent de ne pas être soumis à " la pression des nécessités immédiates " (Millet \& Thin, 2005).

Dans notre population, les jeunes femmes qui ont subordonné leurs choix d'orientation à l'anticipation de leurs futurs rôles familiaux ont toutes grandi dans un milieu social où il était possible d'élaborer des projets à long terme. Deux d'entre elles (Geneviève et Marion) sont issues des classes supérieures, tandis que la troisième (Morgane) appartient à cette fraction de classe " situé(e) entre le haut des classes populaires et le bas des classes moyennes " que Cartier, Coutant, Masclet et alii (2008) nomment les « petits moyens ". Dans tous les cas, leurs deux parents ont des emplois pérennes et des revenus réguliers. Aucun n'a jamais connu de chômage, de temps partiel contraint ou d'incertitude durable sur son avenir professionnel. Signe de cette stabilité, tous ont d'ailleurs pu se lancer dans l'acquisition de leur logement alors que leurs enfants étaient encore petits.

À l'inverse, parmi les enquêtées qui n'ont pas fait leurs choix d'orientation en fonction de cette logique, plusieurs ont vécu dans des contextes familiaux rendant plus difficile l'élaboration de projets à moyen ou long termes. Certaines ont grandi auprès de parents manifestant de manière particulièrement visible cet " hédonisme des classes populaires » qui porte à une forme de décontraction par rapport à l'avenir - dans laquelle on peut voir aussi une forme de fatalisme. On le voit par exemple dans l'extrait suivant, issu de l'entretien avec Bérangère Cotti (père ouvrier en invalidité, mère caissière) : "Mon père, explique Bérangère, peu importe ce que je choisis [comme orientation], il dit toujours: "Y'a pas de sots métiers, patati patata". Mais sans savoir de quoi il parle je pense. Je crois qu'ils [mes parents] sont un peu largués sur la scolarité aujourd'hui. [...] En sortant du lycée j'ai été à la 
fac, donc je suis partie de chez mon père, et après la fac j'ai arrêté, j'ai complètement craqué, et j'ai fait un CAP de peintre en bâtiment. Et j'ai appelé mon père pour lui dire : "Bon ben voilà je fais ça", et il m'a dit : "Oh très bien, y'a pas de sots métiers". Après je lui ai dit: "Je vais aux Beaux-Arts", il m'a dit: "Fais ce que t'as envie, je sais que t'y arriveras". II est comme ça, il me pose pas de questions pour plus tard ».

D'autres enquêtées ont connu pendant de nombreuses années des conditions de vie précaires engendrant une incertitude permanente par rapport à l'avenir et limitant là aussi les possibilités de se projeter dans l'avenir à long terme. Nora Bedjaoui (père ouvrier, mère inactive) a vécu cette situation de manière particulièrement marquée. Au moment des entretiens encore, cette jeune femme (âgée de 23 ans) ne fait aucun projet, même à moyen terme. Elle «profite du chômage » et ne sait pas ce qu'elle fera par la suite, mais cette incertitude ne l'inquiète nullement, signe qu'il s'agit pour elle d'une situation habituelle : " Là je profite, j'ai un an pour partir en voyage, faire des trucs, et puis après on verra bien [...] Peut-être que l'année prochaine je pars, je sais pas où, sur un bateau, n'importe quoi, j'en sais rien ". Sur le plan privé non plus, Nora ne se projette pas dans l'avenir : "Je me dis que si j'ai des enfants, ils viendront quand ils viendront [...] Je fais pas de plans. "

Les conditions de vie que Nora a connues au sein de sa famille ont assurément contribué à façonner cette habitude de vivre au jour le jour. Ses parents ont vécu pendant plusieurs années de petite délinquance, avant que le père ne soit incarcéré pendant quatre ans. À l'issue de cette incarcération, ils partent à l'étranger " en voyage " pendant deux ans, reviennent en France, puis se séparent lorsque Nora est en sixième. Mme Bedjaoui et ses enfants (qui sont au nombre de sept) logent alors dans des foyers, puis dans une maison louée, chez des amis, des membres de la famille et à nouveau dans des foyers, avant de s'installer chez une tante de Nora. En 2000, alors que Nora entre en seconde, ses parents se remettent en couple. La famille déménage à nouveau, et la situation des Bedjaoui se stabilise. L'histoire de Nora est ainsi marquée par des conditions de vie particulièrement instables, peu propices à la projection dans l'avenir. Dans ce contexte, on comprend que cette jeune femme ne se soit pas orientée en fonction d'un projet professionnel et/ou familial à long terme. Au lycée, elle s'inscrit en série $L$ option théâtre parce qu'elle aime cette pratique qu'elle a découverte au collège. Après le bac, elle décide de poursuivre cette formation à l'université, toujours par goût pour le théâtre et sans avoir aucun projet professionnel.

\section{Se préoccuper de l'articulation entre vie familiale et vie professionnelle : l'influence des modèles de division sexuelle du travail domestique et parental}

Subordonner ses projets scolaires et professionnels à ce que l'on anticipe des difficultés liées à la conciliation entre vie familiale et vie professionnelle suppose également que l'on se préoccupe de cette question de la conciliation, autrement dit que l'on ait conscience des difficultés qu'elle peut poser et que l'on se sente concerné par ces difficultés. Dans la construction de cette préoccupation, l'influence des modèles auxquels les jeunes filles ont été exposées au sein de leur famille du point de vue de la division sexuelle du travail domestique et parental s'avère décisive. Les enquêtées qui ont fait leurs choix d'orientation en fonction de ce qu'elles anticipent de leurs futurs rôles familiaux ont grandi dans un environnement familial qui les a amenées à prendre conscience de manière particulièrement aiguë des difficultés liées à la conciliation entre vie familiale et vie professionnelle. Toutes ont en effet une mère active, qui a été confrontée à ces difficultés, et qui l'a été de manière d'autant plus importante que le travail domestique et parental, particulièrement lourd étant donné la taille de leur famille, reposait essentiellement sur elle. Lorsque les pères de ces familles contribuaient à ce travail, c'était seulement à la marge (sortir la poubelle ou accompagner sa femme faire les courses), sur le mode du service rendu, et en se centrant sur les tâches parentales plus que sur les tâches domestiques.

Dans cette situation, les mères de ces jeunes femmes ont éprouvé les difficultés de la conciliation entre vie familiale et vie professionnelle de manière particulièrement importante. Mme Lemaire, par exemple, explique qu'elle a " couru pas mal » pendant toute la période où ses enfants étaient petits (elle en a eu cinq), qu'elle ne prenait pas de pause déjeuner afin de rentrer chez elle plus tôt le soir, qu'elle était « à vingt-deux de tension " dès le matin en raison des multiples tâches ménagères qu'elle effectuait avant même de partir à l'hôpital, qu'elle a souvent failli arriver en retard sur son lieu de travail parce qu'il lui fallait emmener ses enfants à l'école, et qu'elle a "séché plus d'une fois [s]es cheveux au ventilateur de la voiture ". Exposées à ce type de modèle maternel, Morgane, Geneviève et Marion ont été amenées à prendre conscience de manière 
précoce des difficultés que peut poser l'articulation entre vie familiale et vie professionnelle. Geneviève se souvient ainsi d'avoir toujours vu sa mère " débordée » et l'avoir souvent entendue " râler » en raison de ce manque de temps.

Dans cette prise de conscience, le rang de naissance des enquêtées joue un rôle notable. Toutes sont en effet des aînées. Or dans la plupart des familles rencontrées, c'est approximativement au moment où les aînés entrent au collège que la charge de travail domestique et parental qui pèse sur les mères est la plus conséquente. Inversement, quand les benjamins atteignent ces âges-là, cette charge est généralement réduite, les aînés étant plus autonomes et ayant même souvent quitté le domicile familial. En raison de leur position d'aînée, ces enquêtées ont donc pu voir leur mère confrontée à des difficultés particulièrement importantes pour concilier vie familiale et vie professionnelle.

À l'inverse, les jeunes femmes qui n'ont pas subordonné leurs choix d'orientation à l'anticipation des rôles familiaux n'ont pas connu des conditions de socialisation susceptibles de leur faire voir au même degré les difficultés que peut poser cette conciliation. Plusieurs (quatre sur treize) ont eu une mère inactive qui n'a pas été confrontée à ces difficultés. D'autres ont eu une mère active, mais n'ont pas vu leurs parents se répartir le travail domestique et parental de manière aussi inégale que les parents des familles présentées précédemment. Dans la famille d'Emma Denissov par exemple (père instituteur, mère infirmière), la répartition de ce travail est relativement égalitaire. Même si c'est avant tout la mère qui s'occupe du ménage, du linge et des repas, le père contribue lui aussi à ces tâches de manière régulière, notamment lorsque sa conjointe travaille la nuit ou le week-end : "Si je travaille, explique Mme Denissov, et puis que j'ai pas préparé quelque chose, mon mari va le faire. Le repassage, la plupart du temps, c'est moi qui m'en occupe, mais si je travaille, c'est pareil... Là, par exemple, ce week-end, moi, j'ai travaillé, c'est mon mari qui a fait tout le repassage, qui a fait les machines également, donc voilà. En fait, si c'est moi qui suis là, c'est moi qui m'en occupe, et si... voilà, si je dors, c'est [lui] ". À côté de cette contribution au travail domestique, M. Denissov prend également en charge une part importante du travail parental. D'après Emma, c'est souvent lui qui donnait le bain aux enfants quand ceux-ci étaient petits ("C'était à tour de rôle »). C'est également lui qui les couchait et, en lien avec son métier d'instituteur, qui suivait leurs devoirs. Cette relative indifférenciation dans la division du travail domestique et parental n'est pas étrangère à la position sociale de $\mathrm{M}$. et Mme Denissov. Membres des classes moyennes salariées bien dotées en capital culturel, les parents d'Emma appartiennent à l'une des fractions de classe où l'attachement à la norme égalitaire est le plus fréquent, et où de fait les pratiques des couples se rapprochent le plus de cet idéal (Brousse, 1999).

D'autres enquêtées, enfin, n'ont pas vu leur mère confrontée aux difficultés de la conciliation entre vie familiale et vie professionnelle de manière aussi aiguë que Morgane, Geneviève et Marion parce qu'elles sont les benjamines ou les dernières-nées de leur famille. Cette situation particulière des dernièresnées est accentuée lorsque les écarts d'âge sont importants au sein de la fratrie. On le voit par exemple dans le cas d'Héléna Chautard (père jardinier, mère aide-soignante), qui est la benjamine d'une fratrie recomposée de quatre enfants. Ses deux demisœurs, qui ont vingt-et-un et dix-sept ans de plus qu'elle, ont quitté le domicile familial lorsqu'elle avait 7 ans, et son demi-frère, qui est son aîné de dix ans, était largement autonome à ce moment-là. Alors qu'elle appartient objectivement à une fratrie de quatre enfants, cette enquêtée a donc vécu quasiment comme une fille unique durant la majeure partie de son enfance, et elle n'a par conséquent pas vu sa mère confrontée aux difficultés de l'articulation entre le travail professionnel et la "gestion " d'une famille nombreuse.

\section{Pouvoir envisager la maîtrise de son temps de travail : le rôle des conditions de travail des mères}

Choisir son futur métier - et en amont son orientation scolaire - en raison des facilités qu'il offre du point de vue de l'articulation entre vie familiale et vie professionnelle suppose enfin que le fait d'organiser son temps de travail en fonction de sa vie familiale soit une perspective envisageable, qui appartienne au domaine du possible. De ce point de vue aussi, les jeunes femmes qui se sont orientées vers des métiers censés être faciles à concilier avec une vie de famille ont grandi dans des configurations familiales particulières. Leurs mères ont en effet pu décider dans une certaine mesure de la durée et de l'organisation de leur temps de travail. Cette maîtrise relative de leur temps de travail est liée en premier lieu à la nature des professions qu'elles exercent. Mme Joubert et Mme Lemaire appartiennent à la fonction publique. Or, par comparaison avec les salariés du privé, ceux 
du public ont plus souvent la possibilité de travailler à temps partiel de manière choisie (Narcy, Lanfranchi \& Meurs, 2009). Pour Mme Bernier, qui n'est pas fonctionnaire, la possibilité de décider de la durée et de l'organisation de son temps de travail est liée à l'exercice d'un métier - kinésithérapeute - dans lequel la demande de travail était importante quand ses enfants étaient petits (" [À l'hôpital] ils avaient un immense besoin de kinés donc c'est moi qui ai pu négocier mon temps de travail. "). Au-delà de ces caractéristiques de leurs métiers, si ces mères ont pu décider partiellement de la durée et de l'organisation de leur temps de travail, c'est aussi parce que leurs conjoints ont toujours eu des revenus stables, suffisamment élevés pour que la famille puisse se passer d'une partie du salaire maternel.

Là encore, la plupart des autres enquêtées n'ont pas connu de telles conditions de socialisation. Beaucoup ont vécu dans un contexte familial dans lequel la perspective d'organiser son temps de travail en fonction de sa vie familiale était plus difficilement envisageable pour les mères. Dans certains cas, cette situation est liée à la nature des professions maternelles. Les mères de ces jeunes femmes exercent des emplois de service peu qualifiés - caissière, aide-soignante en maison de retraite, femme de ménage dans une société de service - dans lesquels la possibilité de négocier ses horaires est très réduite. Dans d'autres cas, elle résulte du fait que les mères avaient avant tout besoin de gagner de l'argent. Dans la famille Mejri par exemple (père ouvrier invalide, mère employée dans une clinique), le père tombe gravement malade en 1990 et les ressources de la famille reposent donc essentiellement sur l'activité de la mère à partir de cette date. Mme Mejri effectue alors régulièrement des heures supplémentaires et des gardes de nuit et de week-end pour maintenir le niveau de vie de la famille (qui compte six enfants). Avant 1990, alors que son mari travaillait encore, elle cumulait déjà son emploi à la clinique avec un autre emploi le week-end (elle gardait une dame à domicile), là aussi dans le but d'augmenter les ressources de la famille ${ }^{14}$. En raison de la nécessité où elle se trouvait de subvenir seule aux besoins du foyer, Mme Mejri pouvait ainsi difficilement envisager de subordonner l'organisation de son temps de travail à ses contraintes familiales. De surcroît, elle n'attribuait sans doute qu'une valeur secondaire à cette dimension de son activité en regard des revenus que celleci lui assurait. Socialisée dans ce contexte familial, sa fille Nadia accorde d'ailleurs elle-même une grande importance au fait d'avoir un emploi bien rémunéré et elle se soucie plus de cet aspect de son futur métier que de la question des horaires de travail. Interrogée sur les qualités d'un "bon métier » à ses yeux, elle ne mentionne pas la compatibilité avec la vie familiale, mais cite en revanche le salaire : "Du haut de mes petites études, j'ai quand même beaucoup de prétentions [salariales]. Pour moi franchement c'est important, parce qu'on vit dans une société où on a besoin d'argent, qu'on le veuille ou qu'on le veuille pas. Y'a pas de jugement, c'est une réalité. "

La possibilité de décider, même partiellement, de la durée et de l'organisation de son temps de travail n'est pas distribuée de manière égale dans l'espace social. Dans les familles de milieux populaires, où les niveaux de vie sont par définition plus faibles que dans les autres catégories sociales, et où l'autonomie laissée aux salarié-e-s en ce qui concerne leurs horaires de travail est plus fréquemment limitée voire nulle, cette possibilité est souvent réduite. Par conséquent, elle est sans doute souvent difficilement pensable pour les mères et rarement prise en compte par les filles dans l'élaboration de leurs projets d'avenir.

\section{DES CHOIX DE « COMPROMIS »?}

Dire que les filles font des choix de compromis, ce n'est pas dire seulement qu'elles intègrent l'anticipation de leurs futurs rôles familiaux dans l'élaboration de leurs projets d'orientation, mais aussi qu'elles sont prêtes à limiter leurs ambitions professionnelles pour pouvoir exercer un métier qui leur semble compatible avec ces rôles. Or, le fait de s'orienter vers une profession perçue comme facile à concilier avec une vie de famille n'implique pas nécessairement ce type de compromis. Sur notre terrain, ces choix de compromis apparaissent nettement dans les cas de Geneviève Lemaire et de Marion Bernier. En effet, si Geneviève (père radiologue, mère médecin du travail) a choisi de devenir enseignante, elle souhaitait initialement être vétérinaire et elle a renoncé à ce métier parce qu'elle le pensait trop contraignant en termes d'horaires : " Je lui disais [à mon père] : "Je veux avoir des enfants et je me vois mal gérer un travail qui me demande beaucoup de temps, avec des gardes le week-end un peu comme le tien, et en même temps une vie de famille un peu comme maman". " De même, au moment des entretiens, Marion (père chef d'entreprise, mère kinésithérapeute) hésite entre la médecine spécialisée (elle aimerait devenir gynécologue ou gériatre) et la médecine générale, et s'apprête à s'orienter vers la seconde afin d'éviter la contrainte des gardes peu compatible 
de son point de vue avec ses projets familiaux. Dans les deux cas, les choix de ces jeunes femmes se caractérisent bien par le renoncement à des professions lucratives et reconnues socialement, au profit de métiers moins rémunérateurs et moins prestigieux, mais dont les horaires leur paraissent plus faciles à concilier avec une vie de famille.

En revanche, dans le cas de Morgane Joubert, le projet de l'enquêtée (passer le concours de professeur des écoles) ne peut pas être qualifié de " choix de compromis ". Pour cette jeune femme issue du " haut " des classes populaires (son père est ouvrier, sa mère secrétaire), devenir institutrice représente en effet une réelle promotion, à la fois objectivement - aucun de ses parents n'a fait d'études supérieures - et subjectivement - elle explique que pour elle, faire cinq années d'études après le bac est quelque chose de " difficile ", signe qu'il s'agit de son point de vue d'un projet ambitieux. Le choix de cette profession ne résulte donc pas d'un compromis entre ambitions sociales et désir d'exercer un métier perçu comme facile à concilier avec une vie de famille. Il constitue au contraire un moyen de réaliser ces deux objectifs simultanément.

Le fait de subordonner son orientation à l'anticipation de ses futurs rôles familiaux peut ainsi affecter différemment les trajectoires des jeunes filles. Même si nos observations portent sur un petit nombre de cas et ne sauraient être généralisées sans travail empirique supplémentaire, elles suggèrent que ce choix se paye plus souvent d'une limitation des ambitions professionnelles des filles dans les catégories sociales supérieures. Pour les jeunes filles de ces catégories, l'ascension sociale et même la seule reproduction de la position parentale passent en effet nécessairement par l'engagement dans des professions appartenant à la catégorie socioprofessionnelle des cadres et professions intellectuelles supérieures. Or la plupart de ces professions exigent un investissement temporel important (Chenu, 2002) et elles sont donc potentiellement problématiques pour qui souhaite exercer une activité professionnelle dont les horaires sont aisément compatibles avec une vie de famille. À l'inverse, pour les filles de milieux populaires, accéder à une profession où il est possible de choisir ne serait-ce que partiellement la durée et l'organisation de son temps de travail (par exemple et en particulier à une profession qualifiée dans la fonction publique) constitue sans doute plus fréquemment une promotion sociale. Pour cette raison, l'orientation vers ce type de profession peut représenter dans ces catégories non pas un choix de compromis mais au contraire un choix doublement tentant.
L'analyse de nos entretiens montre par ailleurs que même dans les cas où les décisions d'orientation des filles impliquent le renoncement à des professions valorisées, ce renoncement reste limité, borné en quelque sorte par la socialisation de classe. Les choix d'orientation de Geneviève et de Marion ont ainsi été élaborés en fonction d'un horizon des possibles déterminé, celui des destins scolaires et sociaux qui sont envisageables - et souhaitables - au sein de la classe sociale dont elles sont issues (Bourdieu \& Passeron, 1971). Geneviève, qui est issue d'une famille nettement plus dotée en capitaux économiques et scolaires que Morgane, n'a ainsi jamais envisagé de devenir professeure dans le primaire. Lorsqu'elle a décidé de devenir enseignante, c'était pour exercer ce métier à son niveau de qualification et de rémunération le plus élevé, celui de l'agrégation (de manière significative, elle n'a même pas passé le CAPES). Elle indique d'ailleurs que lorsqu'elle a annoncé à sa mère son projet d'être professeure, celle-ci l'a fortement incitée à enseigner en classe préparatoire plutôt qu'au lycée : "Ma mère voulait absolument que je sois prof en prépa. Elle voulait absolument que je fasse un truc grandiose, le top du top ". De même, Marion, qui vient d'une famille de cadres supérieurs du privé (ses parents et ses grands-parents travaillent tous ou ont travaillé dans ce secteur), n'a jamais pensé elle non plus devenir institutrice, ni plus largement enseignante. Elle s'est orientée vers une profession libérale, dans un domaine d'activité (la médecine) dont elle a régulièrement pu entendre parler au sein de sa famille puisque sa mère est kinésithérapeute, son parrain est psychologue après avoir fait des études de médecine, et la femme de celui-ci est dentiste. Dans les deux cas, la socialisation de classe, très précisément l'intériorisation des "espérances subjectives de [leurs] parents ou des adultes les plus significatifs de [leur] entourage " (Lahire, 2010), a joué pour ces jeunes filles un rôle de force de rappel, les empêchant en quelque sorte d'abaisser leurs ambitions scolaires et sociales de manière trop drastique.

\section{CONCLUSION}

La logique du " choix de compromis " apparaît donc au final comme une manière parmi d'autres de décider de son orientation, qui ne concerne pas l'ensemble des filles, loin de là. Même dans notre population de jeunes femmes issues de familles nombreuses, où l'on pouvait s'attendre à ce que ces choix 
soient particulièrement fréquents, la plupart des enquêtées n'ont pas décidé de leur orientation en fonction de cette logique. Ce constat rejoint un certain nombre de résultats de recherche récents. Dans son enquête sur les élèves de l'enseignement professionnel, U. Palheta (2012) signale ainsi que son matériau ne " valide guère " l'idée selon laquelle les choix d'orientation des filles trouveraient leur principe dans l'anticipation de leurs futures contraintes familiales. Lorsque les jeunes filles qu'il a interviewées décrivent comment s'est déroulée leur orientation, elles ne mentionnent en effet nullement cette logique. De même, plusieurs enquêtes statistiques réalisées au cours de ces dix dernières années sur les projets scolaires et professionnels des lycéens montrent que seule une minorité de filles (de l'ordre de 10 à $20 \%$ ) disent vouloir exercer un métier qui leur laisse " suffisamment de temps libre " (Nauze-Fichet, 2005) ou qui leur permette d'" avoir un emploi du temps compatible avec la vie familiale " (Lemaire \& Leseur, 2005).

Ces observations ne signifient évidemment pas que les inégalités entre hommes et femmes dans la répartition du travail domestique auraient disparu, ni que les coûts de la conciliation entre vie familiale et vie professionnelle auraient cessé de peser prioritairement sur les femmes. Elles signifient simplement que subordonner son orientation scolaire et professionnelle à l'anticipation de ses futurs rôles familiaux nécessite des dispositions particulières, associées à des conditions de socialisation qui ne sont pas distribuées de manière uniforme dans l'espace social. En s'appuyant sur les analyses présentées ici, on peut faire l'hypothèse que cette manière de décider de son orientation concerne de manière privilégiée les filles qui appartiennent à certaines fractions des classes moyennes ou supérieures. De manière plus précise, on est amené à penser qu'elle doit être moins fréquente chez les filles des classes moyennes et supérieures bien dotées en capital culturel, où les modèles de division sexuelle du travail domestique sont plus égalitaires qu'ailleurs. Sans doute également s'observe-t-elle moins dans les classes populaires, où les conditions de vie restreignent souvent les possibilités de projection dans l'avenir, et où, en raison de la limitation des ressources et de la nature des métiers féminins, le fait de pouvoir organiser son temps de travail en fonction de sa vie familiale constitue une perspective plus difficile à envisager pour les femmes.

Au-delà de cette discussion de la notion de choix de compromis, nous espérons avoir montré l'intérêt qu'il y a à prêter attention aux effets de la socialisation de classe dans l'analyse des choix d'orientation des filles. En prenant en compte cette dimension de la socialisation, on se donne les moyens d'affiner la connaissance des processus qui sont au principe de ces choix, et on s'évite en particulier la tentation d'attribuer à l'ensemble des filles des conduites qui ne s'observent en fait que chez certaines d'entre elles ${ }^{15}$.

Martine Court martine.court@univ-bpclermont.fr Université Blaise-Pascal-Clermont-Ferrand 2, LAPSCO

Julien Bertrand

Université Paul-Sabatier-Toulouse 3, PRISSHM-SOI et centre Max Weber

Géraldine Bois ENS de Lyon, centre Max Weber

Gaële Henri-Panabière Université Paris-Descartes-Sorbonne Paris Cité, CERLIS

Olivier Vanhée ENS de Lyon, centre Max Weber 
1 En ce qui concerne l'usage du mot " conciliation ", nous adoptons ici la position de Pailhé et Solaz (2010). Tout en rappelant les problèmes que pose ce terme, qui tend à masquer que les coûts de l'articulation entre vie familiale et vie professionnelle pèsent avant tout sur les femmes, ces deux auteures se prononcent en faveur de son utilisation. À l'appui de ce choix, elles expliquent que, dans son usage actuel, ce terme sert moins à désigner un compromis harmonieux entre vie familiale et vie professionnelle qu'à signaler précisément les difficultés de leur articulation, et que d'autres termes, comme celui d'« articulation ", ne permettent pas plus que celui de "conciliation " de dire que ces difficultés incombent avant tout aux femmes.

2 Dans le premier texte publié sur ce sujet, M. Duru-Bellat (1991) parle d'ailleurs de "stratégie " de compromis. Dans les textes postérieurs, elle préférera l'expression « choix de compromis ".

3 De manière significative, cette précision est développée uniquement dans une note de bas de page (Duru-Bellat, 1995, p. 82).

4 Parler de « choix " d'orientation ne doit pas faire oublier que ces choix se font à l'intérieur d'un champ des possibles qui n'est pas également " ouvert " pour tous les élèves et qu'ils sont bien souvent des choix " de nécessité " (Bourdieu, 1979) ou des choix à « modalité unique » (Orange, 2010). Pour une discussion de ce terme, voir Blanchard et Cayouette-Remblière (2011).

5 Cette recherche, réalisée en 2010-2011, a fait l'objet d'un financement par la Caisse nationale des allocations familiales (CNAF).

6 De manière complémentaire, deux autres modes de recrutement ont été utilisés. Quatre familles ont été contactées au moyen d'un questionnaire diffusé auprès d'étudiants inscrits à l'université dans différentes filières d'études (sciences humaines, lettres et sciences). Trois familles ont également été recrutées par l'intermédiaire d'une association d'aide aux populations en difficultés sociales. Ces deux modes de recrutement ont notamment permis un rééquilibrage de notre population en faveur des familles de milieux populaires.

7 Certains entretiens ont ainsi été particulièrement longs (durant parfois jusqu'à trois heures)

8 Notre enquête diffère également de celle de M. Duru-Bellat par le fait qu'elle concerne une population de taille plus réduite.
9 Afin de respecter l'anonymat des personnes interviewées, leurs noms et prénoms ont été modifiés.

10 Les situations de ce type sont assurément moins fréquentes dans notre population d'enquête que dans l'ensemble de la population, dans la mesure où les élèves en difficulté ou en échec scolaire, qui se voient plus souvent que les autres imposer leurs choix d'orientation, sont sous-représentés parmi nos enquêtés.

11 Pour l'institution scolaire, un « vrai » projet d'orientation est « un projet personnel construit sur la base des intérêts de l'individu et de la vision que celui-ci a de son futur " (André, 2012, p. 109).

12 G. André fait référence ici aux écrits de R. Hoggart (1970). Dans le cas de la France, les travaux de P. Bourdieu (1979) ont également mis en évidence l'existence de ce rapport spécifique à l'avenir chez certaines fractions des classes populaires.

13 Dans son travail sur les élèves de l'enseignement professionnel, U. Palheta (2012) observe lui aussi cette " insouciance » chez certains de ses enquêtés (essentiellement les apprentis). II montre cependant que cette attitude ne concerne qu'une partie d'entre eux, d'autres (les élèves des filières tertiaires) percevant au contraire leur avenir avec inquiétude.

14 Réduire cette situation professionnelle à la seule nécessité économique serait bien sûr simplificateur. Si Mme Mejri a ainsi multiplié les heures supplémentaires, c'est aussi parce qu'elle trouvait un certain nombre de gratifications dans son travail : "Quand j'étais au lycée, raconte sa fille Nadia, elle faisait les nuits pour arrondir les fins de mois, et puis elle, elle a besoin un peu de se couper de la maison pour être bien. Elle a trouvé un certain épanouissement, et du coup voilà ça régit son équilibre. " Néanmoins, le poids de ces contraintes économiques ne doit pas non plus être sous-estimé.

15 Nous remercions les rédacteurs en chef de la revue ainsi que les relecteurs anonymes pour les remarques constructives qu'ils nous ont adressées à propos de la première version de ce texte. Nous restons seuls responsables des analyses qui y sont présentées.

\section{BIBLIOGRAPHIE}

ANDRÉ G. (2012). L'orientation scolaire. Héritages sociaux et jugements professoraux. Paris : PUF.

BAUDELOT C. \& ESTABLET R. (1992). Allez les filles ! Paris : Éd. du Seuil.

BERTRAND J., BOIS G., COURT M., HENRI-PANABIÈRE G. \& VANHÉE O. (2012). Les parcours scolaires des enfants de familles nombreuses : l'analyse des processus de socialisation. Rapport adressé à la Caisse nationale des allocations familiales.

BLANCHARD M. \& CAYOUETTE-REMBLIÈRE J. (2011). "Penser les choix scolaires ». Revue française de pédagogie, $\mathrm{n}^{\circ} 175$, p. 5-14.

BLANPAIN N. (2007). " Les conditions de vie des familles nombreuses ». Études et résultats, no 155.

BOUDON R. (1979). La logique du social. Paris : Hachette.

BOURDIEU P. (1979). La distinction. Critique sociale du jugement. Paris : Éd. de Minuit.

BOURDIEU P. (1980). Le sens pratique. Paris : Éd. de Minuit.
BOURDIEU P. \& PASSERON J.-C. (1971). La reproduction. Éléments pour une théorie du système d'enseignement. Paris : Éd. de Minuit.

BOSSE N. \& GUÉGNARD C. (2007). « Les représentations des métiers par les jeunes: entre résistances et avancées ". Travail, genre et sociétés, no 18, p. 27-46.

BROUSSE C. (1999). " La répartition du travail domestique entre conjoints reste très largement spécialisée et inégale ». INSEE-France, portrait social, p. 135-151.

CAILLÉ J.-P., LEMAIRE S. \& VROLANT M.-C. (2002). « Filles et garçons face à l'orientation ». Note d'information du ministère de l'Éducation nationale, no 02-12.

CARTIER M., COUTANT I., MASCLET O. \& SIBLOT Y. (2008). La France des "petits-moyens ». Enquête sur la banlieue pavillonnaire. Paris : La Découverte.

CHENU A. (2002). « Les horaires et l'organisation du temps de travail ". Économie et statistique, no 352-353, p. 151-167.

DUBET F. \& MARTUCELLI D. (1996). À l'école. Sociologie de l'expérience scolaire. Paris : Éd. du Seuil. 
DURU-BELLAT M. (1991). " La raison des filles : choix d'orientation ou stratégies de compromis ? ". L'Orientation scolaire et professionnelle, no 3, p. 257-267.

DURU-BELLAT M. (1995). «Socialisation scolaire et projets d'avenir chez les lycéens et les lycéennes. La "causalité du probable" et son interprétation sociologique ". L'Orientation scolaire et professionnelle, no 1, p. 69-86.

DURU-BELLAT M. (2004). L'école des filles. Quelle formation pour quels rôles sociaux ? Paris : L'Harmattan.

HOGGART R. (1970). La culture du pauvre. Paris : Éd. de Minuit.

LAHIRE B. (2000). “ De la théorie de l'habitus à une sociologie psychologique ». In B. Lahire, Le travail sociologique de P. Bourdieu, dettes et critiques. Paris : La Découverte, p. 121-152.

LAHIRE B. (2010). «La transmission familiale de l'ordre inégal des choses ". Regards croisés sur l'économie, n॰ 7 , p. 203-210.

LEMAIRE S. \& LESEUR B. (2005). « Les bacheliers S : motivations et choix d'orientation après le baccalauréat ". Note d'information du ministère de l'Éducation nationale, no 05-15.

LETURCQ M. \& WIERINK M. (2009). « Temps de travail et bien-être des mères de famille nombreuse ". In A. Pailhé \& A. Solaz, Entre famille et travail, des arrangements de couples aux pratiques des employeurs. Paris : La Découverte, p. 99-120.

MILLET M. \& THIN D. (2005). « Le temps des familles populaires à l'épreuve de la précarité ". Lien social et politiques, no 54, p. 153-162.

NARCY M., LANFRANCHI J. \& MEURS D. (2009). « LeS femmes ont-elles de bonnes raisons d'aller dans le public et l'associatif ? ». In A. Pailhé \& A. Solaz, Entre famille et travail. Des arrangements des couples aux pratiques des employeurs. Paris : La Découverte, p. 363-382.
NAUZE-FICHET E. (2005). « Les projets professionnels des jeunes sept ans après leur entrée au collège ". Éducation et formations, no 72, p. 113-123.

ORANGE S. (2010). " Le choix du BTS. Entre construction et encadrement des aspirations des bacheliers d'origine populaire ". Actes de la recherche en sciences sociales, no 183 , p. 32-47.

ORANGE S. (2012). "Interroger le choix des études supérieures. Les leçons d'un "raté" d'enquête ". Genèses, no 89 , p. 112-127

PAILHÉ A. \& SOLAZ A. (2010). «Concilier, organiser, renoncer : quel genre d'arrangements ? ". Travail, genre et sociétés, no 24, p. 29-46.

PALHETA U. (2012). La domination scolaire. Sociologie de l'enseignement professionnel et de son public. Paris : PUF.

RÉGNIER-LOILIER A. (2006). " L'influence de la fratrie d'origine sur le nombre souhaité d'enfants à différents moments de la vie. L'exemple de la France ». Population, no 61, p. 193-224.

ROCHEX J.-Y. (1992). " Interrogations sur "le projet", la question du sens ". Migrants-Formation, no 89, p. 102118.

ROSENWALD F. (2006). " Filles et garçons dans le système éducatif depuis vingt ans ". INSEE-Données sociales, p. 87-94.

SAINT POL T. de \& BOUCHARDON M. (2013). " Le temps consacré aux activités parentales ». DREES, Études et résultats, no 841.

STEVANOVIC B. \& MOSCONI N. (2007). « La représentation des métiers chez les adolescent(es) scolarisé(es) au collège et au lycée. "Du mouvement mais pas de changement" ». Travail et emploi, no 109, p. 69-80.

van ZANTEN A. (2009). Choisir son école. Stratégies familiales et médiations locales. Paris : PUF.

VOUILLOT F. (2007). " L'orientation aux prises avec le genre ». Travail, genre et sociétés, no 18, p. 87-108. 